\title{
Metformin Inhibits Esophageal Squamous Cell Carcinoma Via- bility and Mobility in vitro through Down-regulation of Long Non-coding RNA CCAT1 and SPRY4-IT1
}

\author{
Min Zhang ${ }^{1,2 x}$, Jixia Wang ${ }^{3 X}$, Yichun Li $^{1}$, Lei Qin ${ }^{4}$, Ruijuan Fan ${ }^{1}$, Yanting Liu ${ }^{1,2}$, Weiwei Li ${ }^{1}$, Yongmei Qin ${ }^{*}$ and \\ Ping $\mathrm{Lu}^{12^{*}}$
}
${ }^{1}$ Department of Oncology, The First Affiliated Hospital of Xinxiang Medical University, Weihui, Henan Prov- ince, P.R. China, 453100; zhangmin1982@xxmu.edu.cn; 498841048@qq.com; fan11666666@163.com; yanting.liu@foxmail.com; 13837384916@163.com; doctorluping@163.com
${ }^{2}$ Life Science Research Center, The First Affiliated Hospital of Xinxiang Medical University, Weihui, Henan Province, P.R. China, 453100.
${ }^{3}$ Department of Oncology, Zhumadian Hospital of Traditional Chinese Medicine, Zhumadian, Henan Prov- ince, P.R. China, 463000; $1490888125 @ q q . c o m$
${ }^{4}$ Department of Gastroenterology, The First Affiliated Hospital of Xinxiang Medical University, Weihui, He- nan Province, P.R. China, 453100; qinlei_soaring@sina.cn; yongmeiqin120@126.com
* Correspondence: e-mail@e-mail.com; Tel.: (optional; include country code; if there are multiple correspond- ing authors, add author initials)
$x$ These authors contributed equally to this work

\begin{abstract}
Evidence indicates that the long noncoding RNAs are involved in the metformin-mediated anti-cancer processes. However, the potential effects of the long noncoding RNAs in metformin-mediated anti-tumor processes in esophageal squamous cell carcinomas (ESCC) are still elusive. This study uncovered that metformin decreases the level of long noncoding RNAs CCAT1 and SPRY4-IT1 thereby contributing to the down-regulation of c-Myc and vimentin. Also, the RNA level test of human ESCC tissue confirmed the positive correlation between CCAT1 and c-Myc. These findings demonstrated that metformin facilitated anti-cancer effects by targeting the 2 long noncoding RNAs (CCAT1 and SPRY4-IT1) and their consequential targets c-Myc and vimentin. Therefore, the CCAT1 and SPRY4-IT1 might act as novel molecular targets that mediate the anti-tumor effects in esophageal squamous cell carcinoma. This helps in predicting the treatment response of metformin in patients diagnosed with esophageal squamous cell carcinoma.
\end{abstract}

Keywords: Esophageal squamous cell carcinoma; Metformin; Long noncoding RNAs; CCAT1; SPRY4-IT1; c-Myc

\section{Introduction}

Based on 2018 reports, there was an estimated 572,034 incidence of esophageal cancer and nearly 508,585 deaths across the globe[1] . Early detection, radical operation, chemoradiotherapy (CRT), and novel approaches including immunotherapy have reduced the incidence and mortality rates of neoplasms since the year 2000. However, esophageal cancer (EC) remains the 3rd leading cause of tumor-related deaths in China[2], specifically, $90 \%$ of the total EC cases are esophageal squamous cell carcinomas (ESCC)[3]. Esophagostomy with/without neoadjuvant therapy is still the golden standard for curative treatment of esophageal carcinoma (ECA). Nevertheless, the long-term survival rate remains unsatisfactory (5-year survival rates range from $20 \%$ to $50 \%$ ) [4, 5] and the recurrence rate is $20 \%$ after radical surgery[5]. Thus, the identification of novel therapeutic strategies for ESCC is urgently necessary. 
Accumulating epidemiological data[6-8] and preclinical investigations[9-12] recently focused on the anti-tumor effects of esophageal cancer (EC) in various tumors. Notably, metformin (1,1-dimethylbiguanide hydrochloride) is a common drug for type 2 diabetes mellitus (T2DM). Based on data from Taiwan's NHI, metformin reduces ECA risk in T2DM patients with cumulative use for more than 2 years[8], consequently drawing on its potential anti-tumor effect in Esophageal cancer.

Research shows that administration of metformin improves the overall survival (OS) and distant metastatic-free survival (DFS) rates in patients with ECA[7]. Pre-clinical studies indicated that metformin sensitizes chemotherapy[13-15] or radiotherapy[16]. Additionally, it induces apoptosis and autophagy[17], inhibits proliferation [18, 19], as well as triggers cell cycle arrest in esophageal cancer [18]. Despite the good anti-cancer effects of metformin, its mechanisms and the clinical impacts in esophageal squamous carcinoma are unclear.

Long noncoding RNAs (lncRNAs)[20] are expressed widely in a various spectrum of neoplasms and promote tumor initiation, progression, invasion, metastasis, and chemo/radiotherapy resistance. Besides, several lines of evidence have revealed its importance in ESCC[21-24]. Nevertheless, the potential roles and mechanisms of lncRNAs implicated in the antineoplastic process of metformin have not been clarified in ESCC.

Herein, we searched the LncRNA from Disease database (http://www.cuilab.cn/lncrnadisease) and identified 7 potential lncRNAs including CCAT1, CCAT2, 91H, ESCCAL-1, ANRIL, SPRY4-IT1, and H19. Further experiments confirmed that CCAT1 and SPRY4-IT1 regulate the anti-cancer mechanism of metformin. Our findings might provide novel molecular targets for ESCC therapy, and an evaluation index for the treatment response of metformin in patients diagnosed with esophageal squamous cell carcinoma.

\section{Results}

\subsection{Metformin inhibited viability and mobility of ESCC cells in a dose-dependent manner}

The effect of metformin on the viability of ESCC cells was assessed by MTT assay. After treatment with different concentrations of metformin $(0,2.5,5,10,20,40 \mathrm{mM})$ for $48 \mathrm{~h}$, the viability of Eca-109 cells was significantly inhibited starting at $5 \mathrm{mM}$, and reduced to less than $50 \%$ of control at $40 \mathrm{mM}$ (Fig. 1A). Also, the Eca-109 cells were treated with metformin in $5 \mathrm{mM}$ and $10 \mathrm{mM}$ for $24 \mathrm{~h}, 48 \mathrm{~h}$, and $72 \mathrm{~h}$. Cell viability decreased to about $80 \%$, $60 \%$, and $48 \%$ after $24 \mathrm{~h}, 48 \mathrm{~h}$, and $72 \mathrm{~h}$ respectively (Fig. 1B). These results suggested that metformin attenuated cell viability in Eca-109 cells in a time- and dose-dependent manner. The inhibition effect of metformin on viability was also observed in another ESCC cell line TE-1 (Fig. 1C).

The wound healing and Transwell assays were performed to investigate the effect of metformin on the mobility of ESCC cells. As shown in Figure 1D and 1E, the mobility of Eca-109 cells was inhibited prominently by metformin even started at the low dose of $1.25 \mathrm{mM}$ (Figure 1E), and the migration efficacy of ESCC cells decreased after treatment with metformin (Fig. 1F, 1G) in a dose-dependent manner.

These data indicate that metformin harbors an inhibitory effect on the growth and migration of ESCC cells.

\subsection{CCAT1 and SPRY4-IT1 were the IncRNAs most significantly affected by metformin in ESCC cells}

Notably, lncRNAs are known to influence the initiation and progression of tumors in a broad spectrum of cancers, including ESCC [24]. To further explore the mechanism of anti-ESCC actions for metformin, a total of 7 lncRNAs (CCAT1, CCAT2, 91H, ESCCAL-1, ANRIL, SPRY4-IT1, and H19) were obtained from the LncRNA and Disease databases (http://www.cuilab.cn/lncrnadisease, until November/20/2015). Real-time PCR was performed to confirm the roles of metformin in the regulation of lncRNAs. After treatment with metformin $(10 \mathrm{mM})$ for $48 \mathrm{~h}, 2$ of 7 lncRNAs i.e., CCAT1 and SPRY4-IT1 were significantly down-regulated in Eca-109 (Fig. 2A) and TE1 (Fig. 2B) cells. Further experiments 
confirmed that the expression of CCAT1 (Fig. 2C) and SPRY4-IT1 (Fig. 2D) was downregulated in a dose-dependent manner in Eca-109 cells.

Based on these findings, CCAT1 and SPRY4-IT1 potentially regulate metformin-induced anti-ESCC effect.

\subsection{Overexpression of CCAT1 and SPRY4-IT1 reversed metformin-induced inhibition of viability and mobility on ESCC cells}

Rescue assays were performed to further analyze the functional relevance of lncRNAs (CCAT1 and SPRY4-IT1) modulation for the antineoplastic roles of metformin. Eca-109 cells were transfected with empty vector (pcDNA3.1), CCAT1-expressing plasmid (CCAT1), or SPRY4-IT1-expressing plasmid (SPRY4-IT1). The RNA levels of CCAT1 and SPRY4-IT1 increased more than 20 fold and 4,000 fold after $48 \mathrm{~h}$ of transfection respectively (Fig. 3A). These data suggested that the viability of Eca-109 cells was significantly promoted after overexpression of CCAT1 or SPRY4-IT1. In addition, a decrease in the viability was observed after treating with metformin but was reversed by co-transfection of CCAT1 or SPRY4-IT1 (Fig. 3B). A similar phenomena were observed in the regulation of mobility (Fig. 3C-E). After transfecting with CCAT1 or SPRY4-IT1, an increase in mobility and migration was observed in Eca-109 cells. Metformin decreased the mobility of Eca-109 cells, and the over-expression of either of the 2 lncRNAs relieved this inhibition.

As a consequence, these data implied that the function of metformin on ESCC cell viability and mobility was at least partially associated with the regulation of CCAT1 and SPRY4-IT1.

\subsection{Metformin inhibited the expression of E-cadherin, vimentin, and c-Myc in ESCC cells}

Recent studies have reported EMT reversal and anticancer effects of metformin in a broad spectrum of neoplasms[16]. Additionally, previous research has demonstrated the regulation of the 3 EMT markers E-cadherin, Vimentin and N-cadherin by metformin and the 2 lncRNAs (CCAT1 and SPRY4-IT1). Further, the regulation of 3 EMT markers (Ecadherin, Vimentin, and N-cadherin) were estimated in ESCC cell lines to elucidate the underlying mechanisms. These data indicate that the protein level of vimentin was significantly downregulated in a dose-dependent manner, and a slight reduction of E-cadherin was also determined in ESCC cells (Fig. 4). There was no apparent difference in the expression of E-cadherin between the metformin-treated and control groups (Fig. 4), Various reports indicate that as a target of metformin, c-Myc also plays a pivotal role in the anti-cancer effects of metformin[10]. Our findings demonstrate that the protein expression of c-Myc was significantly downregulated in Eca-109 (Fig. 4A, B) and TE-1 cells (Fig. 4C, D). Also, when treated with metformin, the protein level of c-Myc was down-regulated in a dose-dependent manner (Fig. 4).

\subsection{Metformin modulated c-Myc and vimentin levels via CCAT1 and SPRY4-IT1}

Reports indicate that CCAT1 and SPRY4-IT1 regulate the expression of EMT markers[26-28] and c-Myc[29]. Thus, pcDNA3.1, CCAT1, or SPRY4-IT1 were overexpressed in Eca-109 cells. The cells were then treated with metformin ( 0 or $10 \mathrm{mM})$ for $24 \mathrm{~h}$. Real-time PCR results revealed the RNA levels of CCAT1 and SPRY4-IT1 in 4 groups (Fig. 5A, B). Western blotting outcomes showed that when CCAT1 was up-regulated, there was a concomitant increment in the protein levels of c-Myc and vimentin, as well as a concomitant up-regulation in expression of E-cadherin proteins. The protein levels of c-Myc and vimentin were downregulated by metformin, which were also rescued by partial over-expression of CCAT1 (Fig. 5C, E). A similar phenomenon was observed in cells transfected with SPRY4-IT1 (Fig. 5D, F).

These data demonstrated that the regulation of vimentin and c-Myc by metformin potentially depends on the partial expression of 1ncRNAs (CCAT1 and SPRY4-IT1). 
2.6. Expression of CCAT1, SPRY4-IT1 and c-Myc in human ESCC tissues and ESCC cell lines Several reports have demonstrated the relationship between c-Myc and CCAT1 in hepatocellular carcinoma (HCC) tissues[29], nonetheless, no research has explored this in human ESCC. Herein, the expression of c-Myc, CCAT1, and SPRY4-IT1 in the 15 pairs of ESCC tissues and matched adjacent normal tissues was evaluated by real-time RT-PCR. Consequently, the outcomes indicated that the CCAT1 levels were up-regulated in ESCC tissues compared to normal tissues (right panel), and the CCAT1 up-regulation rate was 60\% (Figure 6A). Meanwhile, higher levels of SPRY4-IT1 (Fig. 6B, right panel) and c-My (Fig. 6C, right panel) were also found in ESCC tissues compared to normal tissues. The up-regulation rate of SPRY4-IT1 and c-Myc was 80\% (Fig. 6B, left panel) and 80\% (Fig. 6C, left panel), respectively. Furthermore, the correlations between CCAT1/SPRY4-IT1 and cMyc were analyzed. A positive correlation was discovered between the RNA levels of CCAT1 and c-Myc $(r 2=0.306, P \leq 0.05$, Fig. 6D), while no correlation was observed between the expression of SPRY4-IT1 and c-Myc. Also, the expression of CCAT1, SPRY4-IT1, and c-Myc was tested in TE1, Eca-109, TE7, and KYSE150. The data showed a similar expression trend (Fig. 6 F, G) and positive correlation between CCAT1 and c-Myc (Fig. 6 I), but not between SPRY4-IT1 and c-Myc (Fig. 6 F, H, J).

These data suggested that RNA levels of SPRY4-IT1 and CCAT1 were upregulated in ESCC tissues, thereby indicating a positive correlation between CCAT1 and c-Myc in human ESCC tissues and cell lines. 
A

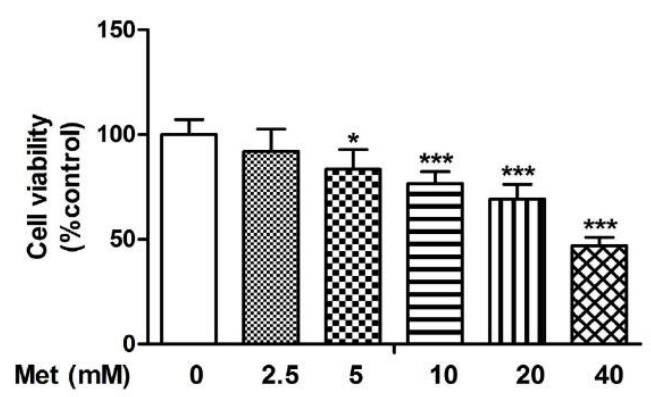

C

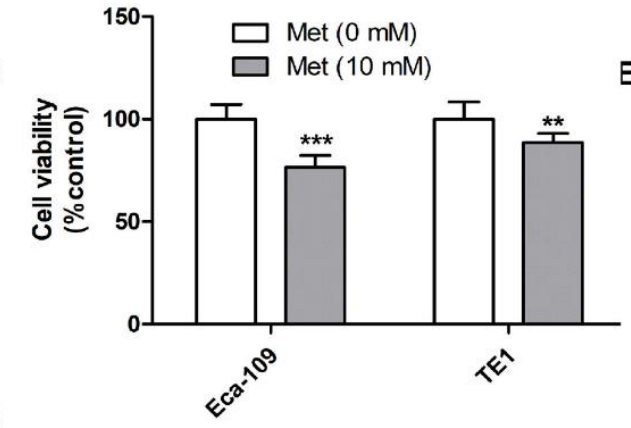

B

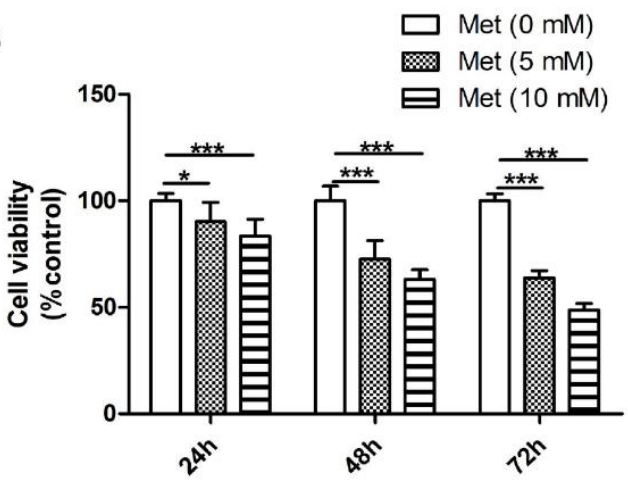

E

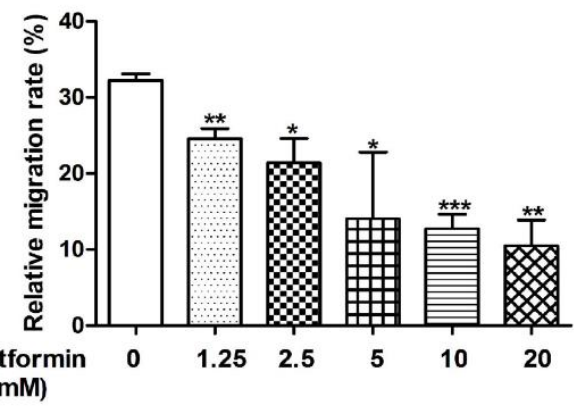

Met (mM)

0

1.25

2.5
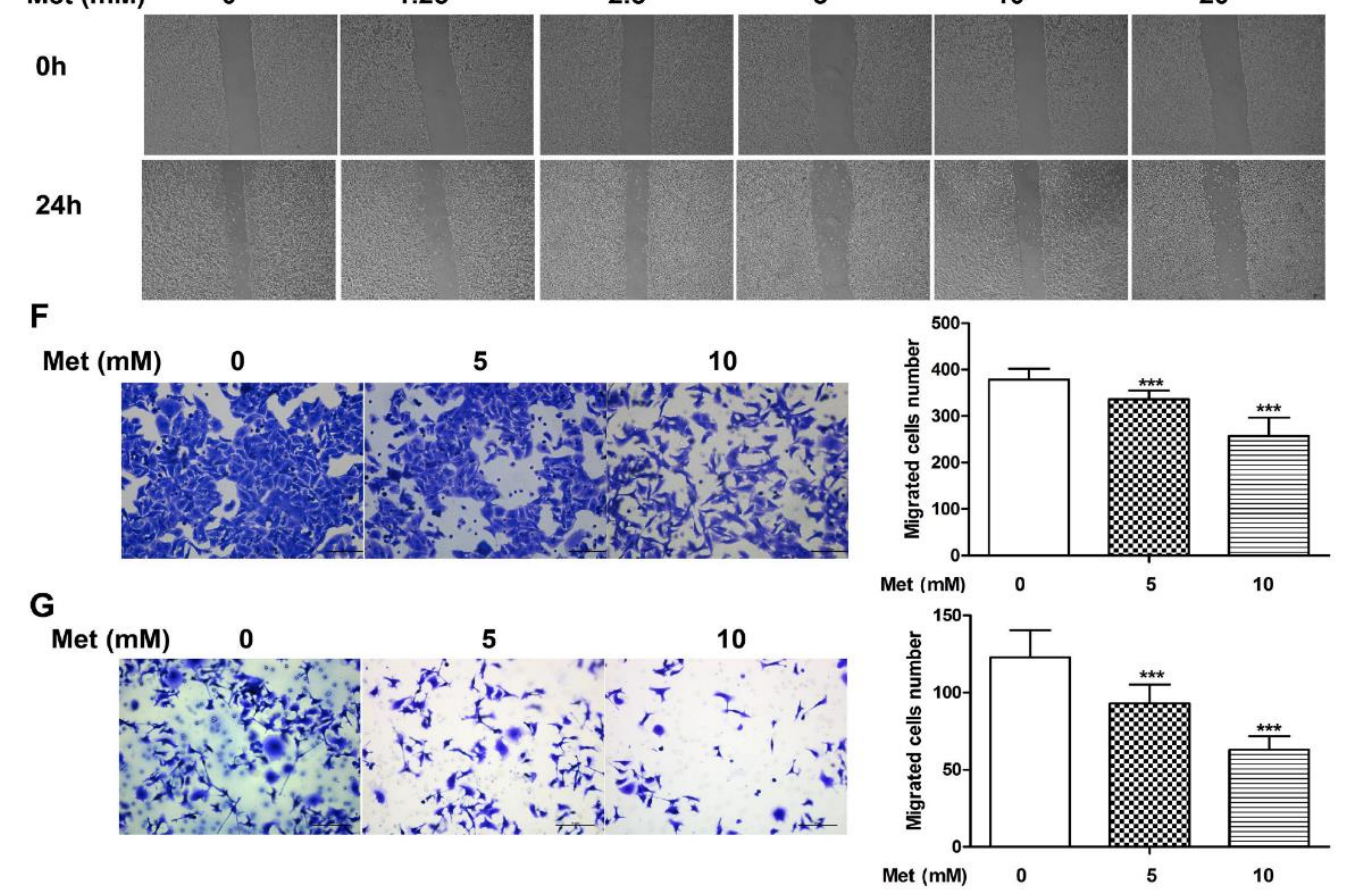

5

10

20

Figure 1. Effects of metformin on the proliferation and mobility of ESCC cells. (A) Cell viability of Eca-109 was detected by MTT assays after treated with metformin (0, 2.5, 5, 10, 20 and $40 \mathrm{mM})$ for 48 h. (B) Cell viability of Eca-109 was detected by MTT assays after treated with metformin (0, 5 and $10 \mathrm{mM}$ ) for $24 \mathrm{~h}, 48 \mathrm{~h}$ and $72 \mathrm{~h}$. (C) ESCC cell lines were treated with metformin $(10 \mathrm{mM})$ for 48 h. (D) Wound healing assay was employed to detect the mobility of Eca-109 cells treated with metformin for $24 \mathrm{~h}$ and $(\mathrm{E})$ wound healing rates were presented by the histograms. The in vitro migration ability of Eca-109 cells (F) and TE-1 cells (G) was determined using Transwell assay. Representative photomicrographs of migrated cells are shown in the left panel, and the number of migrated cells is shown in the right panel. The quantification of cell migration was presented as cell number $\left({ }^{*} \mathrm{P}<0.05,{ }^{* *} \mathrm{P}<0.01,{ }^{* *} \mathrm{P}<0.001\right)$. 


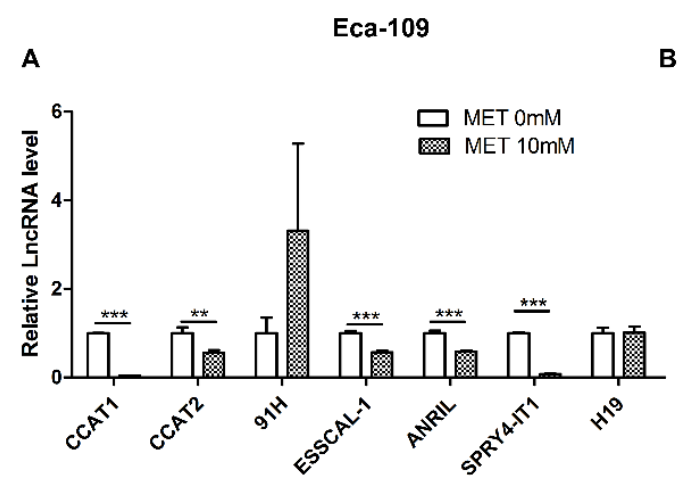

B

TE1

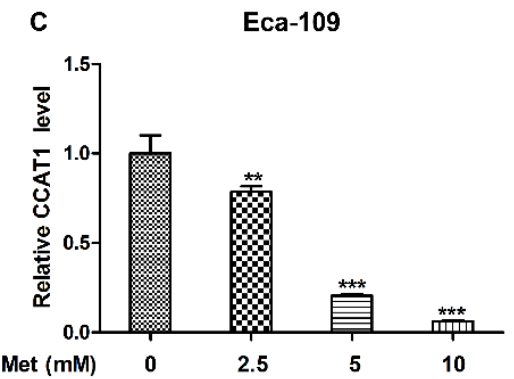

D
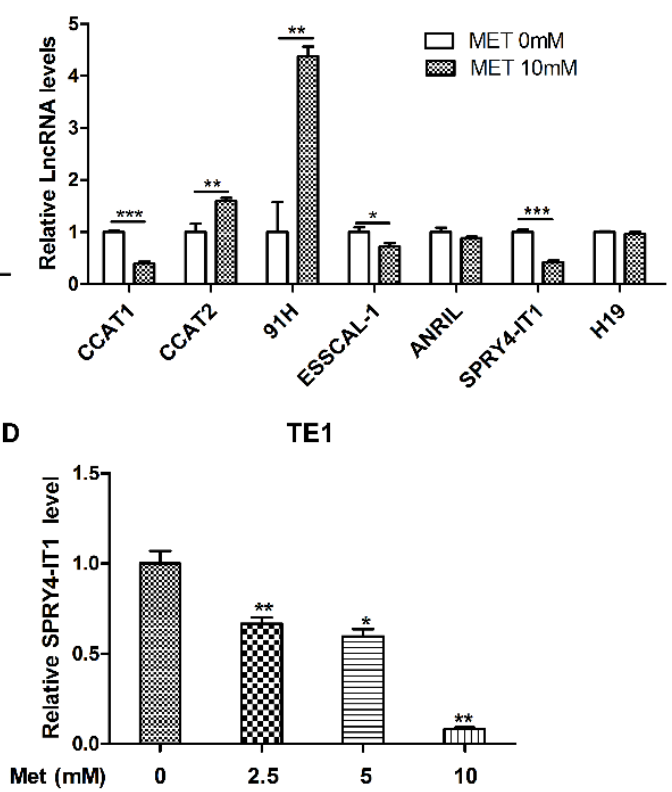

Figure 2. CCAT1 and SRY4-IT1 were the lncRNAs most significantly affected by metformin in ESCC cells. In Eca-109 cells (A) and in TE1 cells (B), the expression of seven LncRNAs was detected by real-time PCR. The level of CCAT1 (C) and SPRY4-IT1 (D) was detected by real-time PCR after treated with metformin $(0,2.5,5$ and $10 \mathrm{mM})$ for $48 \mathrm{~h}$ in Eca-109 cells. ${ }^{*} \mathrm{P}<0.05,{ }^{* *} \mathrm{P}<$ $0.01,{ }^{* * *} \mathrm{P}<0.001$. 


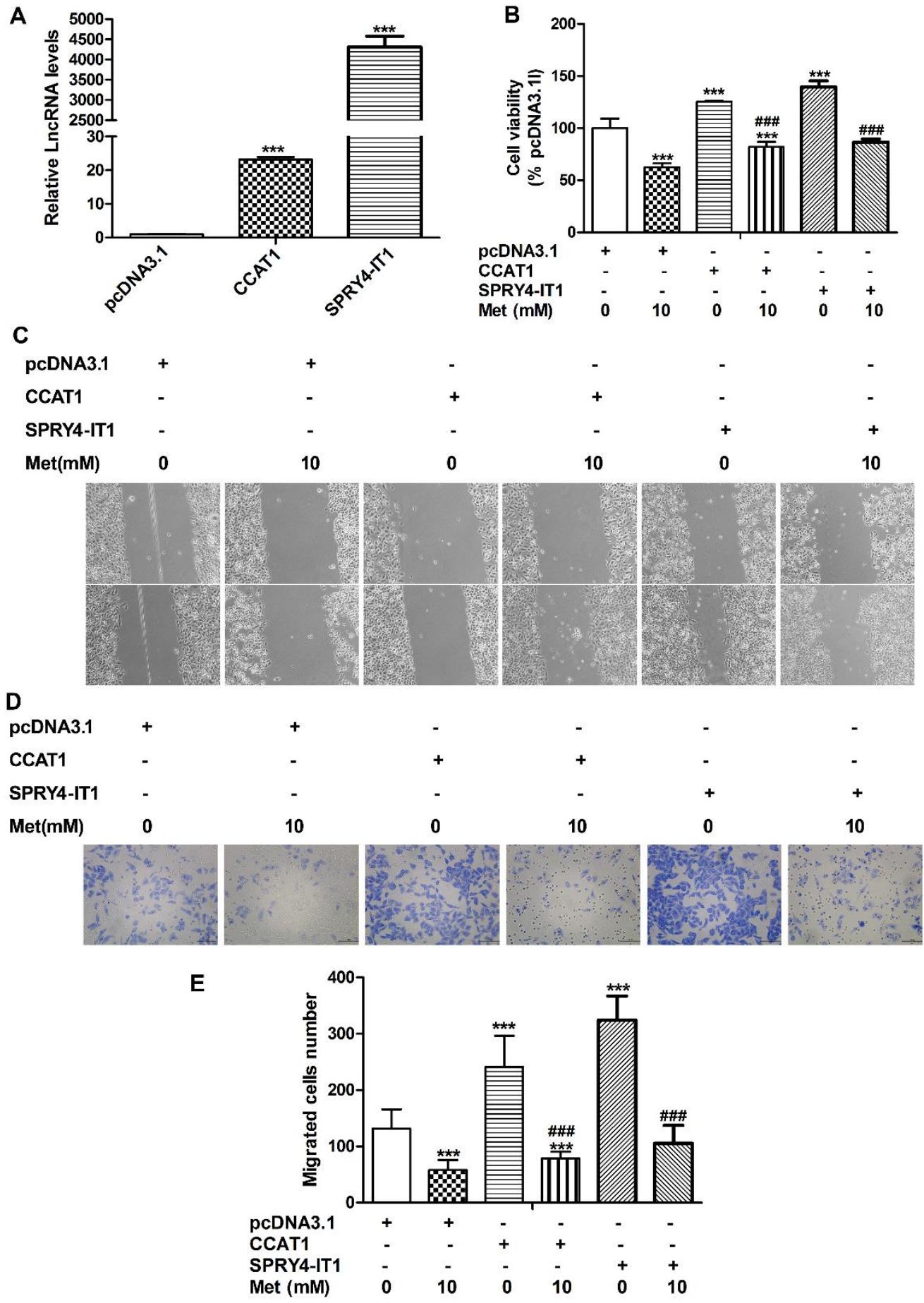

Figure 3. LncRNAs CCAT1 and SPRY4-IT1 mediated the anticancer effects of metformin. Eca-109 cells were transfected with empty vector (pcDNA3.1), CCAT1-expressing plasmid (CCAT1) or SPRY4-IT1-expressing plasmid (SPRY4-IT1) . (A) RNA levels were analyzed by real-time RT-PCR $48 \mathrm{~h}$ post transfection. (B) Cell viability was analyzed $48 \mathrm{~h}$ post transfection. (C) Wound healing assay and (D) Transwell assay were employed to measure the mobility of Eca-109 cells $24 \mathrm{~h}$ post transfection. (E) Histograms showing the number of migrated cells ( ${ }^{* * *} \mathrm{P}<0.001 \mathrm{vs}$. pcDNA3.1 group, \#\# $<0.001$ vs. pcDNA3.1+Met group). 
A
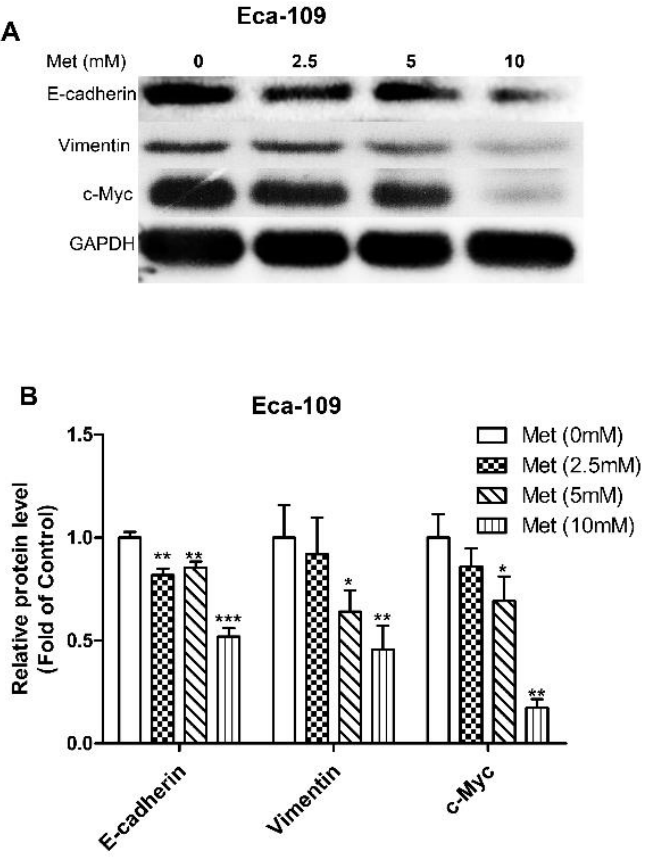

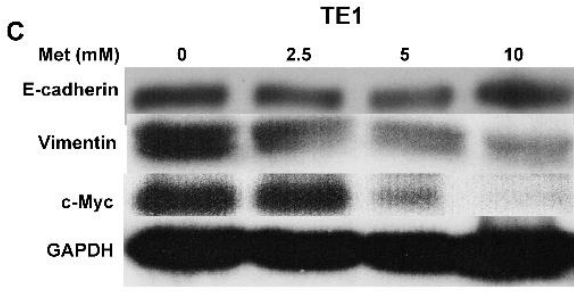

D

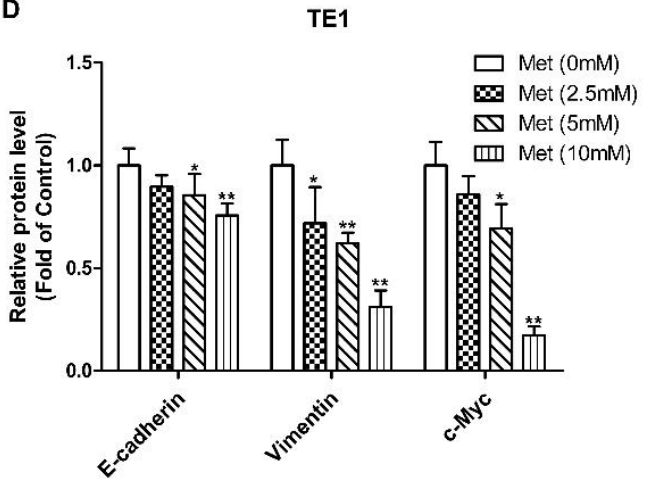

Figure 4. Expression of E-cadherin, Vimentin and c-Myc in metformin-treated ESCC cells. ESCC cells were treated with different concentrations of metformin $(0,2.5,5,10 \mathrm{mM})$ for $48 \mathrm{~h}$. Western blotting was done to detect the protein levels of E-cadherin, Vimentin and c-Myc in Eca-109 cells (A) and TE1 cells (C), and quantified by densitometry (B, D) $\left({ }^{*} \mathrm{P}<0.05, \quad{ }^{* *} \mathrm{P}<0.01,{ }^{* * *} \mathrm{P}<0.001\right)$. 
A

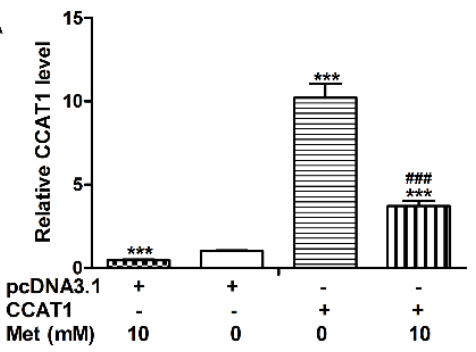

C

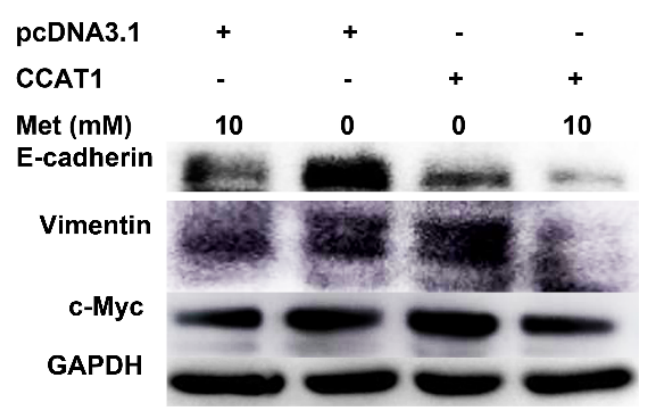

$\mathbf{E}$

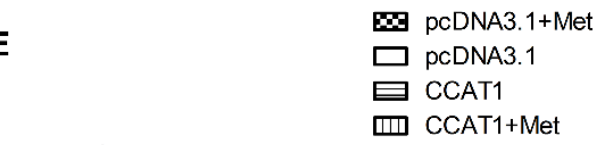

B

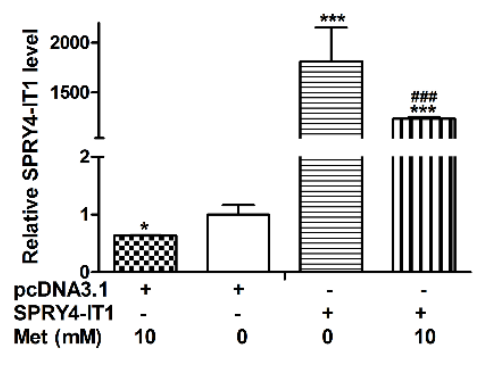

D

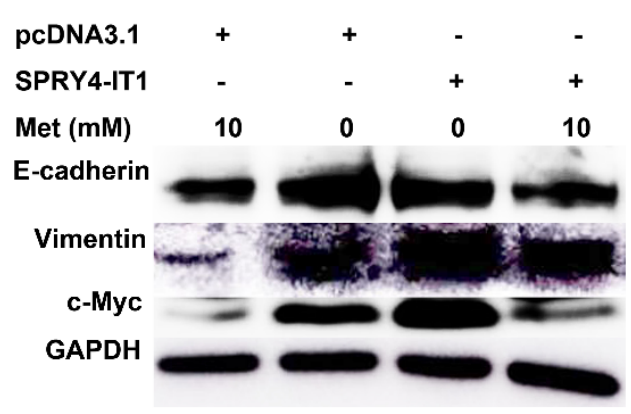

$\mathbf{F}$

B pcDNA3.1+Met

$\square$ pcDNA3.1

品PRY4-IT1

四 SPRY4-IT1+Met

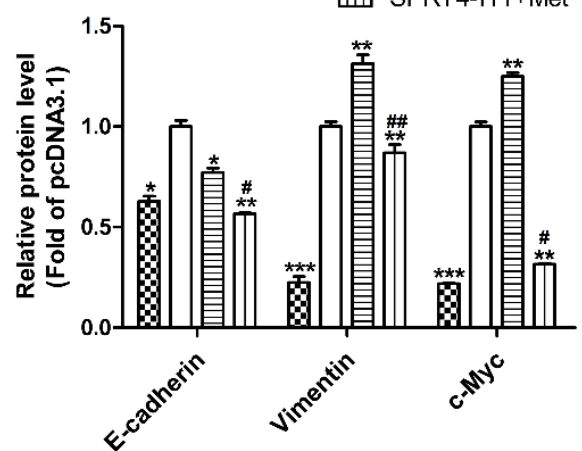

Figure 5. Metformin modulated c-Myc and Vimentin levels via CCAT1 and SPRY4-IT1. Eca-109 cells were transfected with empty vector (pcDNA3.1), CCAT1-expressing plasmid (CCAT1) or SPRY4-IT1-expressing plasmid (SPRY4-IT1) . Real-time PCR analysis showed the (A) CCAT1 and (B) SPRY4-IT1 RNA levels of Eca-109 cells. Western blotting assays examined the protein expression of c-Myc, Vimentin and E-cadherin in Eca-109 cells transfected with CCAT1 (C) or SPRY4-IT1 (D) respectively, and quantified by densitometry (E, F) $\left({ }^{*} \mathrm{P}<0.05,{ }^{* *} \mathrm{P}<0.01,{ }^{* * *} \mathrm{P}<0.001\right.$ vs. pcDNA3.1 group. \# $\mathrm{P}<0.05$, \#\# $\mathrm{P}<0.01$, \#\#\# $\mathrm{P}<0.001$ vs. pcDNA3.1 + Met group). 

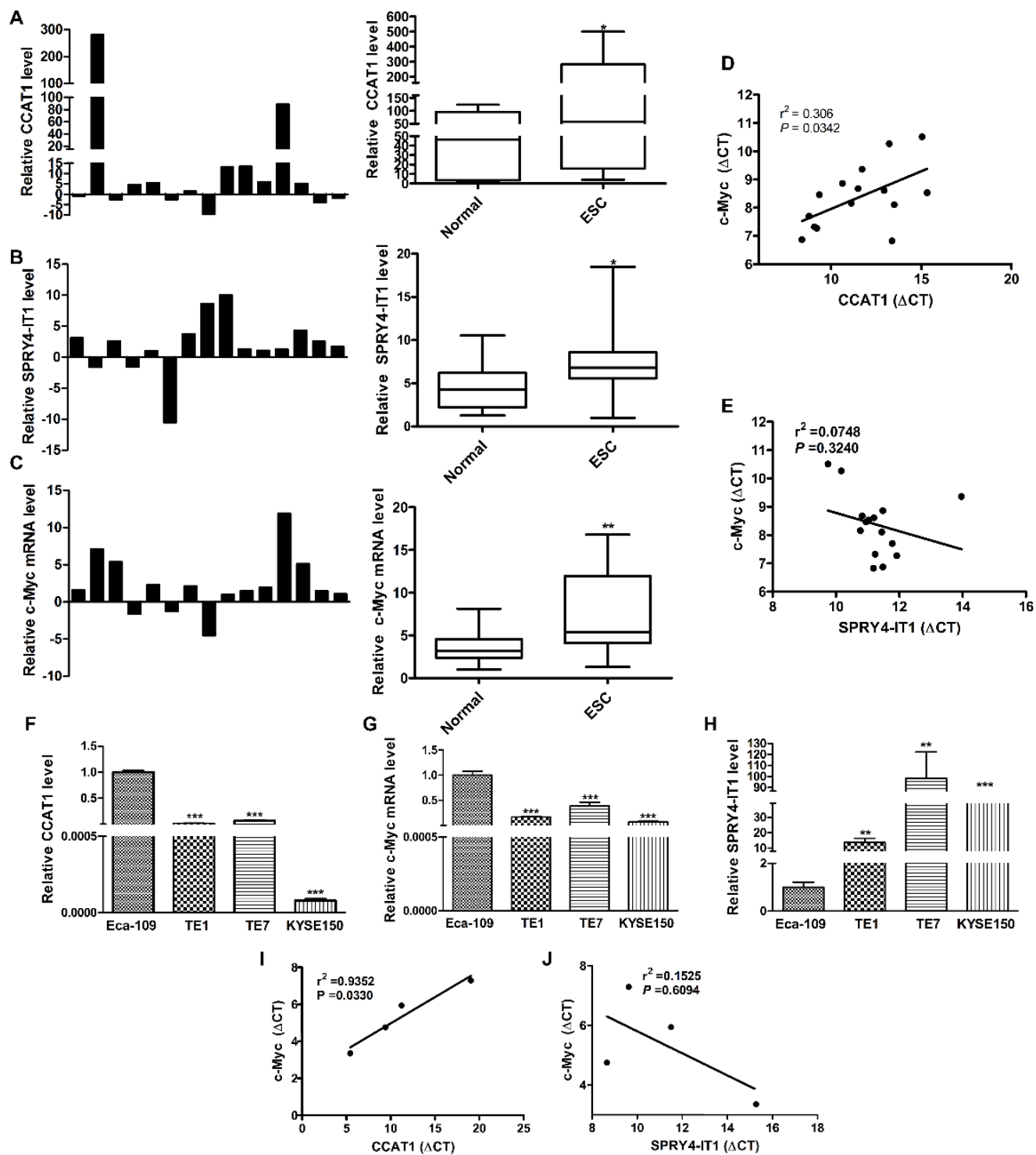

Figure 6. Relative expression of c-Myc CCAT1 and SPRY4-IT1 in human ESC tissues compared with adjacent normal tissues. The levels of CCAT1, SPRY4-IT1 and c-Myc were examined by realtime RT-PCR, normalized to $\beta$-actin expression, and were calculated by $2-\Delta C T$. (A-C) Relative expression of CCAT1, SPRY4-IT1 and c-Myc in ESCC tissues $(n=15)$ compared with corresponding adjacent normal tissues (normal) $(n=15)$, and the results are presented as fold changes in tumor tissues relative to compared normal tissues (left panels). Relative CCAT1, SPRY4-IT1 and cMyc levels were significantly increased in ESCC tissues (with respect to lowest one of each group, CCAT1: Normal2, SPRY4-IT1 ESCC6, c-Myc: ESCC12) (right panels). (D) The correlation between CCAT1 level and c-Myc mRNA level was measured in 15 ESCC tissues. (E) The correlation between SPRY4-IT1 level and c-Myc mRNA expression was measured in 15 ESC tissues. (F - H) Relative expression of CCAT1, SPRY4-IT1 and c-Myc in 4 ESCC cell lines. (I) The correlation between CCAT1 level and c-Myc mRNA level was measured in 4 ESCC cell lines. (J) The correlation between SPRY4-IT1 level and c-Myc mRNA expression was measured in 4 ESCC cell lines. Relative levels were subjected to Pearson correlation analysis ( ${ }^{*} \mathrm{P}<0.05, \quad{ }^{* *} \mathrm{P}<0.01,{ }^{* * *} \mathrm{P}<0.001$ ). 


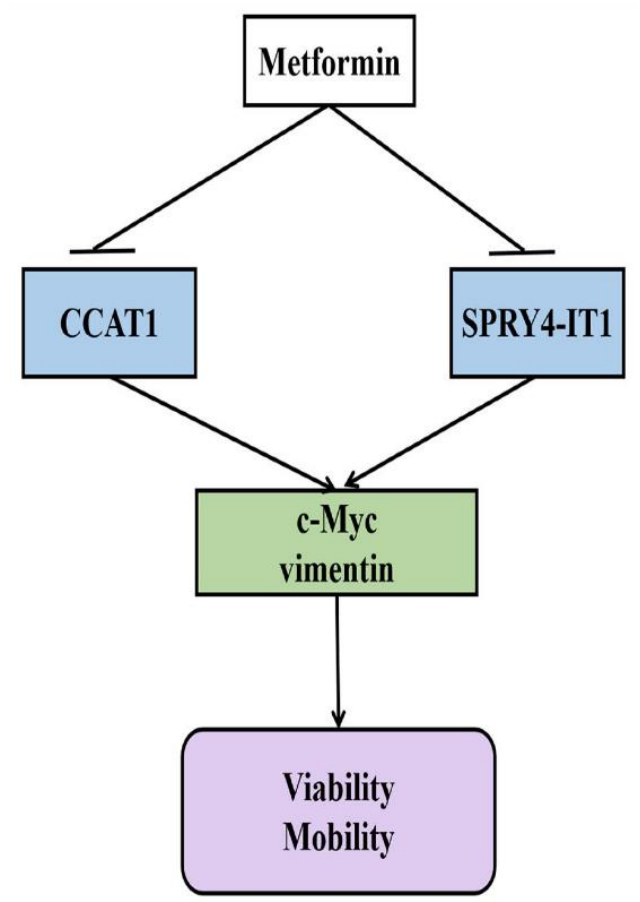

Figure 7. A schematic model of the proposed mechanisms for anti-cancer effects of metformin in ESCC cells. Metformin down-regulates the CCAT1/ SPRY4-IT1 and consequential c-Myc and vimentin to inhibits the viability and mobility of ESCC cells in vitro.

\section{Discussion}

The incidence and mortality rates of ESCC have dramatically increased across the globe during the past decade, specifically in China[1-3]. Despite the emergence of many novel strategies in the past few years, their outcomes have not been satisfactory. Therefore, it is urgent to identify new, cost-effective, and safe therapeutic targets for ESCC therapy. In the past decades, metformin has been confirmed to be valuable in esophageal cancer patients[6-8]. For instance, it demonstrated the efficient anti-viability effect of metformin on ESCC cells $[14,17,18]$. Also, this work demonstrated that metformin inhibited the viability and migration of ESCC cells in a dose- and time-dependent manner (Figure 1). Nonetheless, the precise mechanism still needs to be explored.

LncRNAs regulate genes in the transcription process particularly in cancer biology. Besides, their various functions in the regulation of cancer has become an area of research focus in recent years. Our study revealed that metformin treatment significantly downregulates 2 lncRNAs (CCAT1 and SPRY4-IT1) in ESCC cells (Figure 2), up-regulates these 2 lncRNAs (CCAT1 and SPRY4-IT1) as well as promotes the viability and mobility of Eca109 cells in vitro (Figure 3). Moreover, the rescue assays confirmed metformin-induced suppression of viability and migration in vitro by targeting CCAT1 and SPRY4-IT1 (Figure 3). Further studies indicated that c-Myc and vimentin might participate in this process. Conclusively, our study found that Metformin might promote the anti-viability and antimobility effects of ESCC cells by modulated c-Myc and vimentin levels, which was regulated by CCAT1 and SPRY4-IT1(Fig. 7). These findings further clarify the anti-cancer mechanism of metformin and give highlights that CCAT1 and SPRY4-IT1 might act as a novel target for ESCC therapy.

\section{Materials and Methods}

\section{Cell culture}

A total of 4 human ESCC cell lines TE-1, Eca-109, EC9706, and KYSE-150 were purchased from the Cell Bank of Institute of Biochemistry and Cell Biology of the Chinese Academy of Sciences (Shanghai, China). TE-7 was provided by Dr. Ruili Sun (Department 
of Laboratory Medicine, Xinxiang Medical College). First, TE-1, EC9706, KYSE-150 and TE-7 cells were cultured in RPMI-1640 (SH30809.01, HyClone) containing 10\% fetal bovine serum (FBS, Gibco-BRL, Gaithersburg, MD). Eca-109 cells were maintained in Dulbecco's modified Eagle medium (DMEM)/high glucose (SH30022.01, HyClone) containing 10\% FBS. Notably, the mediums mentioned above were supplemented with $100 \mathrm{U} / \mathrm{mL}$ penicillin and $100 \mathrm{mg} / \mathrm{mL}$ streptomycin. The cells mentioned above were cultured in a humidified incubator containing $5 \% \mathrm{CO} 2$ at $37^{\circ} \mathrm{C}$.

\section{Reagents}

Metformin (S1741), crystal violet staining solution (C0121), and the methylthiazolyldiphenyl-tetrazolium bromide (MTT, ST316) were purchased from Beyotime Institution of Biotechnology (Nanjing, China). Compound C (S7840) and AICAR (S1802) were purchased from Selleck (Shanghai, China). c-Myc (ab32072) was purchased from Abcam Biotechnology (Cambridge, Massachusetts, USA), GAPDH (CW0101) was purchased from CW Biotechnology (Cambridge, Massachusetts, USA), E-cadherin (\#3195), p-AMPK (\#2535), vimentin (\#5741) and N-cadherin (\#13116) were purchased from Cell Signaling Technology (Danvers, Massachusetts, United States). The RNAiso Plus RNA isolation reagent (9108), PrimeScript ${ }^{\mathrm{TM}} \mathrm{RT}$ reagent Kit with gDNA Eraser (RR047A), and SYBR® Premix Ex Taq ${ }^{\mathrm{TM}}$ (Tli RNaseH Plus) Kit (RR420A) were obtained from TAKARA Biotechnology CO. LTD (Dalian, China).

\section{Cell viability assay}

Cell viability was analyzed using the MTT assay based on the manufacturer's protocols [25]. Cells were plated in 96-well plates at a density of $8 \times 104$ cells $/ \mathrm{ml}$ (for Eca-109), $4 \times 104$ cells $/ \mathrm{ml}$ (for TE1), $3 \times 104$ cells $/ \mathrm{ml}$ (for TE7) or $3.5 \times 104$ cells $/ \mathrm{ml}$ (for EC9706) in a final volume of $100 \mu \mathrm{L}$ of culture medium per well. After treating with vehicle (PBS) or metformin as indicated concentration $(0 \mathrm{mM}, 5 \mathrm{mM}, 10 \mathrm{mM})$ for $24 \mathrm{~h}, 48 \mathrm{~h}$, or $72 \mathrm{~h}$, a total of $20 \mu \mathrm{L}$ $5 \mathrm{~g} / \mathrm{L}$ MTT was added to each well and incubated at $37^{\circ} \mathrm{C}$ for $4 \mathrm{~h}$. After removing the culture mediums containing MTT, a total of $200 \mu \mathrm{L}$ DMSO was added per well to dissolve the formazan crystals. An absorbance microplate reader (SpectraMax Plus 384, Molecular Devices CO. LTD, Shanghai, China) was used to quantify the viable cells by reading the optical density units of absorbance of the supernatants at $570 \mathrm{~nm}$ (OD570). Thereafter, cell viability (\% of control) was calculated as follows: (OD570 of test sample/ OD570 of control) $\times 100 \%$ where 6 wells were measured for each treatment group.

\section{Wound healing assay}

Eca-109 cells were plated into 6-well plates and maintained in DMEM-H with 10\% FBS. After reaching the confluence rate of $90 \%-100 \%$, cells were wounded by scraping with $200 \mu \mathrm{L}$ pipette tips. Eca-109 cells were transfected with pcDNA3.1 (Vector), pcDNA3.1-CCAT1 (CCAT1), or pcDNA3.1-SPRY4-IT1 (SPRY4-IT1). After the confluence rate reached $90 \%$ - 100\%, cells were wounded by scraping with $200 \mu \mathrm{L}$ pipette tips then washed 3 times by PBS. Afterwards, the cells were incubated in a regular medium containing PBS or metformin as indicated concentration for $24 \mathrm{~h}$. Cells were photographed under the microscope (Axio Observer A1, ZEISS) at 0 and $24 \mathrm{~h}$, and the wound width of the scratch was calculated. A total of 3 independent assays were assayed.

\section{In vitro migration assays}

In vitro migration was measured through in vitro Transwell assays. Of note, this study used 24-well Transwell polycarbonate membranes inserted with $8.0-\mu \mathrm{m}$ pores (Costar, Cambridge, MA, USA). Then, 10×104 cells (Eca-109, Eca-109-pcDNA3.1, Eca-109CCAT1 or Eca-109-SPRY4-IT13.1) or 3×104 cells (TE1) were plated in the upper chambers in $0.2 \mathrm{~mL}$ medium without FBS, and the lower chambers were filled with $0.5 \mathrm{~mL}$ medium having 20\% FBS. Medium in both upper and lower chambers were supplemented with PBS or metformin as indicated concentration $(0 \mathrm{mM}, 5 \mathrm{mM}, 10 \mathrm{mM})$. After incubation for $24 \mathrm{~h}$, cells retaining on the upper side of the membrane were erased gently with cotton 
swabs prewetted. The migration cells on the lower surface were fixed in $4 \%$ paraformaldehyde overnight, then stained with crystal violet staining solution for 60 minutes followed by 3 times washing with ddH2O. The number of cells in 9 randomly selected microscopic fields per well (20×) was counted under the Nikon ECLIPSE 55i microscope.

\section{Cell transfection}

The overexpressed vectors of lncRNA i.e., pcDNA3.1-CCAT1 (CCAT1) and pcDNA3.1-SPRY4-IT1 (SPRY4-IT1) (pcDNA3.1/empty vector as control) were synthesized by Bioword (Nanjing, China). Then, the cells were transfected using Opti-MEM I and Lipofectamine2000 reagents (Invitrogen, CA, USA) based on the manufacturer's instructions. After being transfected with the vectors mentioned above, cells were incubated at regular condition for $24 \mathrm{~h}$. For MTT assays, the cells were administrated with metformin for $48 \mathrm{~h}$, and treated with metformin for $24 \mathrm{~h}$ for subsequent experiments.

\section{RNA isolation, reverse transcription, real-time PCR}

Total RNA was extracted using the RNAiso Plus RNA isolation reagent (9108). Reverse transcription was administrated by a PrimeScript ${ }^{\mathrm{TM}} \mathrm{RT}$ reagent Kit with gDNA Eraser (RR047A). Subsequently, the cDNA products were subjected to real-time PCR using a SYBR® Premix Ex Taq ${ }^{\mathrm{TM}}$ (Tli RNaseH Plus) Kit (RR420A). Where, a real-time PCR was performed using a Real-Time PCR instrument (QuantStudio DX, Applied Biosystems) with the amplification program: $95^{\circ} \mathrm{C}$ for $30 \mathrm{~s}$, followed by 40 cycles of $95^{\circ} \mathrm{C}$ for $5 \mathrm{~s}$ and $60^{\circ} \mathrm{C}$ for $34 \mathrm{~s}$. Notably, each sample was analyzed in triplicate. $\beta$-actin mRNA was quantified as an endogenous control. Expressions were calculated as $\Delta \Delta \mathrm{Ct}(2-\Delta \mathrm{Ct})$, and normalized to endogenous controls. The primer sequences are shown in Table 1.

\section{Western blotting}

The experimental procedure applied was based on previous studies [25]. In brief, total proteins were isolated using protein lysis buffer supplemented with PMSF (Beyotime Institution of Biotechnology) and protease inhibitor (Amresco). Protein samples were analyzed by $10 \%$ SDS-PAGE (Beyotime Institution of Biotechnology) and transferred to NC membranes (Pall Gelman Laboratory). For immunodetection, blots were incubated overnight at $4^{\circ} \mathrm{C}$ with primary antibodies. Subsequently, the membranes were washed and incubated with horseradish peroxidase-conjugated anti-rabbit IgG (H+L) (1:5000, Zhongshan Goldenbridge Biotechnology Co., LTD) as a secondary antibody for $1 \mathrm{~h}$. Immobilon Western Chemilum HRP Substrate (WBKLS00100, Millipore Co.) was applied to enable visualization of the bands, then the signals were observed using the Amersham Imager $600 \mathrm{Gel}$ Imaging System (GE Healthcare Life Science). To evaluate the protein levels, the optical densities of the bands in each blot were analyzed using the Image J Analysis System.

\section{cDNA microarray}

The human ESCC cDNA microarrays were obtained from Shanghai Outdo Biotech Co., Ltd. (cDNA-HEsoS030PG01), which comprised 15 ESCC tissues and paired adjacent non-carcinoma tissues. The RNA levels of CCAT1, SPRY4-IT1, and c-Myc were analyzed using RT Real-time PCR as described above.

\section{Statistical analysis}

The data were presented as the mean \pm standard deviation (SD). Statistical analysis was performed using SPSS (Statistical Package for the Social Sciences) (version 21.0). The statistical analyses comparing pairs of groups were performed using the Student's t-test or the two-sample t-test. Multiple group comparisons were analyzed using one-way ANOVA. Linear correlations between 2 variables were analyzed by Pearson's correlation coefficient (r2) (Pearson chi-squared test). The threshold for statistical significance was set at $\mathrm{P} \leq 0.05$.

\section{Conclusions}


Here, we identified a novel role of the 2 lncRNAs (CCAT1 and SPRY4-IT1) in metformin-mediated anti-tumor processes. This indicates that CCAT1 and SPRY4-IT1 potentially serve as an indicator in predicting the anti-cancer efficacy of metformin in patients diagnosed with esophageal cancer.

Author Contributions: M.Z. performed the experiments, J.W. generated and analyzed the data; Y.Q. and P.L. designed experiments, YT.L. revised the manuscript; Y.L., L.Q., R.F. and W.L. provided assistance in materials, experiments, and manuscript preparation. All authors approve the version of the manuscript to be published.

Funding: This work was funded in part by the Medical Science and Technology Project of Henan Province (No. 201702124, No. 201702123), the Joint Fund Project of Xinxiang Medical University (N0. py2014001, N0. py2014006), and the Incubation Found of Xinxiang Medical university (No. 2013QN110).

Institutional Review Board Statement: Not applicable.

Informed Consent Statement: Not applicable.

Acknowledgments: We are grateful to Dr. Ruili Sun (Department of Laboratory Medicine) for providing the TE-7 ESCC cell line. We would also like to thank Dr. Jiateng Zhong (Department of Pathology) and Dr. Zhiwei Feng (College of life science and technology) for their revision of the paper. The 3 doctors mentioned above are all working in Xinxiang Medical University (Xinxiang, China).

Conflicts of Interest: The authors declare that there is no conflict of interest.

\section{References}

1. Bray, F.; Ferlay, J.; Soerjomataram, I.; Siegel, R. L.; Torre, L. A.; Jemal, A., Global cancer statistics 2018: GLOBOCAN estimates of incidence and mortality worldwide for 36 cancers in 185 countries. CA: a cancer journal for clinicians 2018, 68, (6), 394-424.

2. Chen, W.; Zheng, R.; Baade, P. D.; Zhang, S.; Zeng, H.; Bray, F.; Jemal, A.; Yu, X. Q.; He, J., Cancer statistics in China, 2015. Ca A Cancer Journal for Clinicians 2016, 66, (2), 115-132.

3. Torre, L. A.; Bray, F.; Siegel, R. L.; Ferlay, J.; Lortet-Tieulent, J.; Jemal, A., Global cancer statistics, 2012. Ca A Cancer Journal for Clinicians 2015, 65, (2), 87-108.

4. Tachibana, M.; Kinugasa, S.; Yoshimura, H.; Shibakita, M.; Tonomoto, Y.; Dhar, D. K.; Nagasue, N., Clinical outcomes of extended esophagectomy with three-field lymph node dissection for esophageal squamous cell carcinoma. American Journal of Surgery 2005, 189, (1), 98-109.

5. Hirst, J.; Smithers, B. M.; Gotley, D. C.; Thomas, J.; Barbour, A., Defining cure for esophageal cancer: analysis of actual 5-year survivors following esophagectomy. Annals of Surgical Oncology 2011, 18, (6), 1766-1774.

6. Skinner, H. D.; Mccurdy, M. R.; Echeverria, A. E.; Lin, S. H.; Welsh, J. W.; O'Reilly, M. S.; Hofstetter, W. L.; Ajani, J. A.; Komaki, R.; Cox, J. D., Metformin use and improved response to therapy in esophageal adenocarcinoma. Acta Oncologica 2013, 52, (5), 1002-9.

7. Van, D. V. L.; Janssen, L.; Larue, R.; Houben, R.; Buijsen, J.; Sosef, M.; Vanneste, B.; Schraepen, M. C.; Berbee, M.; Lambin, P., Can metformin improve 'the tomorrow' of patients treated for oesophageal cancer? European Journal of Surgical Oncology the Journal of the European Society of Surgical Oncology \& the British Association of Surgical Oncology 2015, 41, (10), 1333-9.

8. Tseng, C. H., Metformin and esophageal cancer risk in Taiwanese patients with type 2 diabetes mellitus. Oncotarget 2016, 8, (12), 18802-18810.

9. Javeshghani, S.; Zakikhani, M.; Austin, S.; Bazile, M.; Blouin, M. J.; Topisirovic, I.; St-Pierre, J.; Pollak, M. N., Carbon source and myc expression influence the antiproliferative actions of metformin. Cancer Research 2012, 72, (23), 6257-67.

10. Blandino, G.; Valerio, M.; Cioce, M.; Mori, F.; Casadei, L.; Pulito, C.; Sacconi, A.; Biagioni, F.; Cortese, G.; Galanti, S., Metformin elicits anticancer effects through the sequential modulation of DICER and c-MYC. Nat Commun 2012, 3, (3), 865-876.

11. Akinyeke, T.; Matsumura, S.; Wang, X.; Wu, Y.; Schalfer, E. D.; Saxena, A.; Yan, W.; Logan, S. K.; Li, X., Metformin targets cMYC oncogene to prevent prostate cancer. Carcinogenesis 2013, 34, (12), 2823-2832.

12. Yan, L.; Zhou, J.; Gao, Y.; Ghazal, S.; Lu, L.; Bellone, S.; Yang, Y.; Liu, N.; Zhao, X.; Santin, A. D., Regulation of tumor cell migration and invasion by the H19/let-7 axis is antagonized by metformin-induced DNA methylation. Oncogene 2014, 34, (23), 3076-3084.

13. Honjo, S.; Ajani, J. A.; Scott, A. W.; Chen, Q.; Skinner, H. D.; Stroehlein, J.; Johnson, R. L.; Song, S., Metformin sensitizes chemotherapy by targeting cancer stem cells and the mTOR pathway in esophageal cancer. International Journal of Oncology 2014, $45,(2), 567-574$.

14. Wang, F.; Ding, X.; Wang, T.; Shan, Z.; Wang, J.; Wu, S.; Chi, Y.; Zhang, Y.; Lv, Z.; Wang, L., Metformin inhibited esophageal squamous cell carcinoma proliferation in vitro and in vivo and enhanced the anti-cancer effect of cisplatin. PloS one 2017, 12, (4), e0174276. 
15. Li, P. D.; Zhao, L.; Tian, T. C.; Wen, G. L.; Jing, Y.; Jing, C.; Zhen, W. Z.; Li, L. C.; Ma, C.; Xiao, F. D., Redox-dependent modulation of metformin contributes to enhanced sensitivity of esophageal squamous cell carcinoma to cisplatin. Oncotarget 2017, 8, (37), 62057-62068.

16. Nakayama, A.; Ninomiya, I.; Harada, S.; Tsukada, T.; Okamoto, K.; Nakanuma, S.; Sakai, S.; Makino, I.; Kinoshita, J.; Hayashi, H., Metformin inhibits the radiation-induced invasive phenotype of esophageal squamous cell carcinoma. International Journal of Oncology 2016, 49, (5), 1890-1898.

17. Feng, Y.; Ke, C.; Tang, Q.; Dong, H.; Zheng, X.; Lin, W.; Ke, J.; Huang, J.; Yeung, S. C.; Zhang, H., Metformin promotes autophagy and apoptosis in esophageal squamous cell carcinoma by downregulating Stat3 signaling. Cell Death \& Disease 2014, 5, (2), e1088.

18. Tang, J. C.; An, R.; Jiang, Y. Q.; Yang, J., Effects and Mechanisms of Metformin on the Proliferation of Esophageal Cancer Cells in vitro and in vivo. Cancer Research \& Treatment 2017, 49, (3), 778-789.

19. Yi, Y.; Jin, G.; Liu, H.; Liu, K.; Zhao, J.; Chen, X.; Wang, D.; Bai, R.; Xiang, L.; Jang, Y., Metformin inhibits esophageal squamous cell carcinoma-induced angiogenesis by suppressing JAK/STAT3 signaling pathway. Oncotarget 2017, 8, (43), 74673-74687.

20. Fatica, A.; Bozzoni, I., Long non-coding RNAs: new players in cell differentiation and development. Nature Reviews Genetics 2014, 15, (1), 7-21.

21. Chen, D.; Zhang, Z.; Mao, C.; Zhou, Y.; Yu, L.; Yin, Y.; Wu, S.; Mou, X.; Zhu, Y., ANRIL inhibits p15(INK4b) through the TGF $\beta 1$ signaling pathway in human esophageal squamous cell carcinoma. Cellular Immunology 2014, 289, (1-2), 91-96.

22. Shahryari, A.; Rafiee, M. R.; Fouani, Y.; Oliae, N. A.; Samaei, N. M.; Shafiee, M.; Semnani, S.; Vasei, M.; Mowla, S. J., Two novel splice variants of SOX2OT, SOX2OT-S1, and SOX2OT-S2 are coupregulated with SOX2 and OCT4 in esophageal squamous cell carcinoma. Stem Cells 2014, 32, (1), 126-134.

23. Xie, H. W.; Wu, Q. Q.; Zhu, B.; Chen, F. J.; Ji, L.; Li, S. Q.; Wang, C. M.; Tong, Y. S.; Tuo, L.; Wu, M., Long noncoding RNA SPRY4-IT1 is upregulated in esophageal squamous cell carcinoma and associated with poor prognosis. Tumour Biol 2014, 35, (8), 7743-7754.

24. Zhang, E.; Han, L.; Yin, D.; He, X.; Hong, L.; Si, X.; Qiu, M.; Xu, T.; De, W.; Xu, L., H3K27 acetylation activated-long non-coding RNA CCAT1 affects cell proliferation and migration by regulating SPRY4 and HOXB13 expression in esophageal squamous cell carcinoma. Nucleic Acids Research 2017, 45, (6), 3086-3101.

25. Zhang, M.; Liu, Y.; Feng, H.; Bian, X.; Zhao, W.; Yang, Z.; Gu, B.; Li, Z.; Liu, Y., CD133 affects the invasive ability of HCT116 cells by regulating TIMP-2. American Journal of Pathology 2013, 182, (2), 565.

26. Sun, M.; Liu, X. H.; Lu, K. H.; Nie, F. Q.; Xia, R.; Kong, R.; Yang, J. S.; Xu, T. P.; Liu, Y. W.; Zou, Y. F., EZH2-mediated epigenetic suppression of long noncoding RNA SPRY4-IT1 promotes NSCLC cell proliferation and metastasis by affecting the epithelialmesenchymal transition. Cell Death \& Disease 2014, 5, (6), e1298.

27. Shen, F.; Cai, W. S.; Feng, Z.; Chen, J.; Feng, J.; Liu, Q.; Fang, Y.; Li, K.; Xiao, H.; Cao, J., Long non-coding RNA SPRY4-IT1 pormotes colorectal cancer metastasis by regulate epithelial-mesenchymal transition. Oncotarget 2016, 8, (9), 14479-14486.

28. Chen, J.; Zhang, K.; Song, H.; Wang, R.; Chu, X.; Chen, L., Long noncoding RNA CCAT1 acts as an oncogene and promotes chemoresistance in docetaxel-resistant lung adenocarcinoma cells. Oncotarget 2016, 7, (38), 62474-62489.

29. Liang, D.; Yang, S. B.; Xu, F. F.; Zhang, J. H., Long noncoding RNA CCAT1 promotes hepatocellular carcinoma progression by functioning as let-7 sponge. Journal of Experimental \& Clinical Cancer Research 2015, 34, (1), 18-27 